\title{
A Method of Flexible Micro-wire Electrode Insertion in Rodent for Chronic Neural Recording and a Device for Electrode Insertion
}

\begin{tabular}{|r|l|}
\hline Journal: & Transactions on Neural Systems \& Rehabilitation Engineering \\
\hline Manuscript ID & TNSRE-2018-00550.R2 \\
\hline Manuscript Type: & Paper \\
\hline Author: & $\mathrm{n}$ /a \\
\hline Complete List of Authors: & $\begin{array}{l}\text { Arafat, Muhammad; Purdue University, Electrical and Computer } \\
\text { Engineering } \\
\text { Rubin, Logan; Purdue University, Biomedical Engineering } \\
\text { Jefferys, John; University of Oxford, Department of Pharmacology } \\
\text { Irazoqui, Pedro; Purdue University, Biomedical Engineering; Purdue } \\
\text { University, Electrical and Computer Engineering }\end{array}$ \\
\hline
\end{tabular}

\section{SCHOLARONE \\ Manuscripts}




\title{
A Method of Flexible Micro-wire Electrode Insertion in Rodent for Chronic Neural Recording and a Device for Electrode Insertion
}

\author{
Muhammad A. Arafat, Logan N. Rubin, John G. R. Jefferys, and Pedro P. Irazoqui, Member, IEEE
}

\begin{abstract}
Reliable chronic neural recording from focal deep brain structures is impeded by insertion injury and foreign body response, the magnitude of which is correlated with the mechanical mismatch between the electrode and tissue. Thin and flexible electrodes cause less glial scarring and record longer than stiff electrodes. However, insertion of flexible microelectrodes into the brain has been a challenge. Here, a novel insertion method is proposed, and demonstrated, for precise targeting deep brain structures using flexible micro-wire electrodes. The microelectrode is spun and slowly inserted in the brain through an appropriate electrode guide. The electrode guide does not penetrate into cortex. Based on two new mechanisms, namely spinning and guided insertion, we have demonstrated successful insertion of 25 micron platinum flexible electrodes about 10 millimeter deep in rat brain without buckling. We present an electrode insertion device based on the proposed method and demonstrate its use to implant flexible microelectrodes in rat brains. The step-by-step insertion process is described. Microelectrodes were inserted in the Bötzinger complex of 11 rat brains and chronic respiratory neural activity was recorded from 2 rats for 50 days.
\end{abstract}

Index Terms-Neural electrode, electrode insertion, electrode buckling, critical buckling strength, electrode insertion device.

\section{INTRODUCTION}

$\mathbf{N}$ EURAL electrodes are a vital tool for brain research and neural prostheses. Neuroscientists use implantable electrodes such as micro-wires and multichannel probes to acquire neural signal from the brain [1]-[5]. Action potentials from specific neurons are recorded to study their behavior [6], [7]. Analyzing neural signals is important for discovering specific mechanisms of brain functions and for investigating brain diseases such as Parkinson's disease, epilepsy and chronic pain [8]-[11]. For chronic sensing, implanted electrodes need to work reliably over time. However, due to the persistent and progressive scarring caused by the foreign body response to electrodes, the number of neurons near the electrode decreases with time [12]. Neural probes made of rigid materials suffer from mechanical mismatch between stiff electrodes and soft

M. Arafat is with the Department of Electrical and Computer Engineering, Purdue University, West Lafayette, IN 47907, USA, e-mail: marafat@purdue.edu.

L. Rubin is with the Department of Biomedical Engineering, Purdue University, West Lafayette, IN 47907, USA.

J. Jefferys is with the Department of Pharmacology, University of Oxford, Oxford, UK and with the Department of Biomedical Engineering, Purdue University, West Lafayette, IN 47907, USA.

P. Irazoqui is with the Departments of Biomedical Engineering and Electrical and Computer Engineering, Purdue University, West Lafayette, IN 47907, USA, e-mail: pip@purdue.edu. brain tissue. Due to the mechanical mismatch and insertion damage, the immune system creates a sheath of astrocytes between the electrode and neurons that moves the neurons away from the recording site over time [13]-[17]. Moreover, the relative micromotion between the brain and electrode creates more glial scar encapsulating the probe and results in neuronal death [18]. Thin and flexible electrodes result in much reduced scar formation [19], [20]. Because of the smaller cross-sectional area, thin electrodes displace a smaller volume of tissue and cause less compression of the surrounding tissue than rigid electrodes. However, as the electrodes become thinner and more flexible, they become more prone to buckle, making insertion more difficult. Whether an electrode will buckle or not depends on the stiffness of the electrode. Critical buckling force $(\mathrm{CBF})$ is a measure of stiffness, which is defined as the maximum force an electrode can withstand before buckling. CBF depends on the geometry of the electrode and the elastic modulus of the electrode material [21]. For a cylindrical electrode, CBF is calculated from Euler's Column Formula as follows [22], [23]:

$$
P_{c r}=\frac{K \pi^{3} E r^{4}}{4 L^{2}}
$$

where, $P_{c r}$ is the CBF of the electrode in Newtons, $K$ is the effective length factor (dimensionless) which depends on the conditions of the end supports of the electrode, $E$ is the elastic modulus of the material in Pascals, $L$ is the effective length in meters and $r$ is the radius of the electrode in meters. The electrodes used in this study are made of 25 micron $(\mu \mathrm{m})$ diameter platinum $(\mathrm{Pt})$ wire because $\mathrm{Pt}$ is widely used for neural electrode fabrication. The elastic modulus of $\mathrm{Pt}$ is 168 GPa. The electrode insertion in brain is generally modeled as 'one-pinned end and one-fixed end' mode of insertion, where $K$ is 2.045. Therefore, from Equation (1), the CBF of a 25 $\mu \mathrm{m}$ diameter Pt electrode of length $16 \mathrm{~mm}$ is $254 \mu \mathrm{N}$, which means that the electrode can only apply $254 \mu \mathrm{N}$ force on the brain surface. However, penetration force experienced by a rigid electrode while penetrating a rat brain is in the range of $500-1000 \mu \mathrm{N}$ provided the dura is removed in advance [24][26]. Therefore, the CBF of the electrode needs to be larger than $1000 \mu \mathrm{N}$ if the dura is removed. Moreover, the required penetration force can be as high as $10 \mathrm{mN}$ if the dura is not removed in advance. Therefore, without increasing the CBF of the electrode or reducing the penetration force of the brain, it is not possible to insert the microelectrode in the brain. One solution to increase CBF is to increase $r$. Doubling $r$ gives 


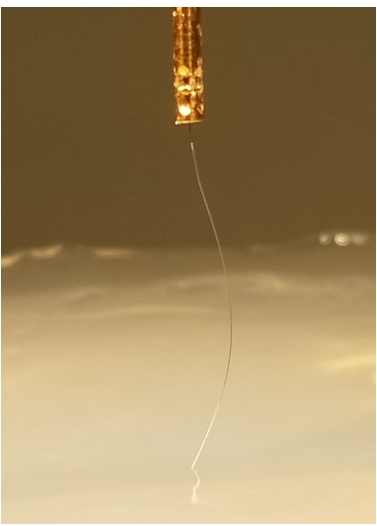

(a)

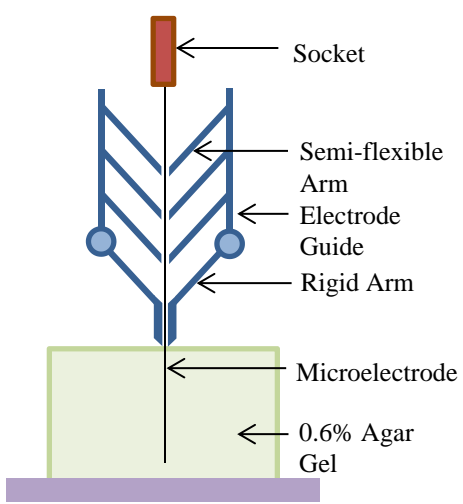

(b)
Fig. 1: (a) Buckling of Pt microelectrode during insertion in 0.6\% agar gel; (b) Proposed electrode guide.

16 times increase in CBF. To have $1000 \mu \mathrm{N}$ CBF required to penetrate into cerebral cortex without dura, the diameter of the electrode has to be $35.2 \mu \mathrm{m}$. To penetrate dura, the diameter has to be $62.6 \mu \mathrm{m}$. However, increasing $r$ makes the electrode stiffer, which is not desirable. Thinner Pt wires, such as $25 \mu \mathrm{m}$ $\mathrm{Pt}$ wire, are available in the market but the insertion of these thin and flexible wire in the brain even with dura removed is a challenge.

In this paper, a method of inserting flexible micro-wire electrodes in the brain with dura is proposed. The method is derived from the principles governing the buckling strength of electrodes. Two new concepts are introduced to increase the $\mathrm{CBF}$ of the electrode and reduce brain penetration force. An electrode insertion device based on the proposed mechanism is presented. In-vivo experiments demonstrate the effectiveness of the proposed insertion mechanism. The ultimate goal of this study is to develop and validate a technology platform that is capable of implanting flexible micro-wire electrodes into deep brain structures for chronic brain studies.

\section{METHOD}

To develop the insertion method, we used $0.6 \%$ agar gel [27]. A $16 \mathrm{~mm}$ long $25 \mu \mathrm{m} \mathrm{Pt}$ microelectrode buckles during insertion in agar gel as shown in Fig. 1a. The proposed electrode insertion method deploys two mechanisms, namely guided insertion and spinning during insertion, to increase the force at which buckling occurs and to penetrate through dura, if it is present, respectively. Conventional insertion uses a stiff rod, cannula or support as a guide which is attached to the flexible electrode with biodegradable materials and the guide is inserted along with the electrode and then removed after insertion [28]-[30]. Our proposed method differs from the conventional, in that the guide stays above the brain, which avoids tissue damage caused by the inserted guide. One candidate for a guide could be a cylindrical tube, but it is problematic because the electrode gets stuck inside the tube when adhesive blood and cerebrospinal fluid (CSF) quickly fill the narrow tube by capillary action during in-vivo insertion experiments. Moreover, retracting the tube after insertion is a problem because the electrode socket cannot pass through the tube. Even if the electrode is detached from the socket before retracting the tube, the tube displaces the electrode while retracting because of the growing friction developed by blood and CSF with time. In addition, attaching the socket to the electrode after insertion is very difficult. To overcome all these problems, we propose a new electrode guide which is shown in Fig. 1b.

The electrode guide is designed to reduce contact area between the electrode and the guide in order to reduce friction due to blood and CSF. The guide consists of 4 pairs of separated arms and each pair forms a micro-hole through which the electrode passes. The guide restricts the electrode from buckling at points where the micro-holes are located. Since the electrode can only buckle between two micro-holes, the effective length of buckling depends on the separation of arms. The guided insertion creates a twofold increase in CBF. First, the guide reduces the effective length of the electrode allowed for buckling. Since CBF is proportional to $1 / L^{2}$, the guide significantly increases CBF. Second, by using a guide, the buckling follows 'both ends fixed' mode rather than 'one-pinned end and one-fixed end' mode when the electrode penetrates cortex [22]. When the electrode is inside brain, the mode of buckling for the portion of electrode above the brain is still in 'both ends fixed' mode. In this mode, $K=4$ which further doubles the CBF. According to Equation (1), a guide having $5 \mathrm{~mm}$ separation between arms can increase the CBF of Pt microelectrode to $5087 \mu \mathrm{N}$, which is large enough to penetrate rat brain if the dura is removed in advance. Therefore, we designed the guide with $5 \mathrm{~mm}$ separation between arms. If the dura is not removed, the brain penetration force can become as high as $10 \mathrm{mN}$. In that case, the electrode is spun slowly to pierce the dura. The spinning electrode bores a micro-hole in the dura and penetrates through it. Even after penetration, the dura sticks to the side wall of the flexible thin wire and clutches the electrode. Therefore, the electrode is spun slowly during insertion until it nears the target. When the electrode is close to the target, we stop spinning. We then advance the electrode in micro-steps into the brain until it reaches the target. The electrode guide is described in section II-B in detail.

\section{A. Microelectrode Fabrication}

The $25 \mu \mathrm{m}$ Pt micro-wire is obtained from A-M Systems, Sequim, WA, USA (Model: 764500). We first take a long piece of wire and carefully straighten it by gently pulling it along the length by fingers. Then we cut the straight wire into 17 $\mathrm{mm}$ long pieces. Each $17 \mathrm{~mm}$ long piece of wire is used to make one electrode. In this study, the insertion depth is about $10 \mathrm{~mm}$. Among the additional $7 \mathrm{~mm}$ length of wire, $6 \mathrm{~mm}$ is required by the design of the electrode inserter and $1 \mathrm{~mm}$ is used to attach a metallic socket to the electrode to hold it during insertion. A gold plated female socket, obtained from Plastics1, Roanoke, VA, USA (Model: E363/0), is attached to one end of the bare wire using a wire crimper. The length of the wire becomes $16 \mathrm{~mm}$. The wire is then insulated by coating with parylene $\mathrm{C}$. The socket is covered with silicone 


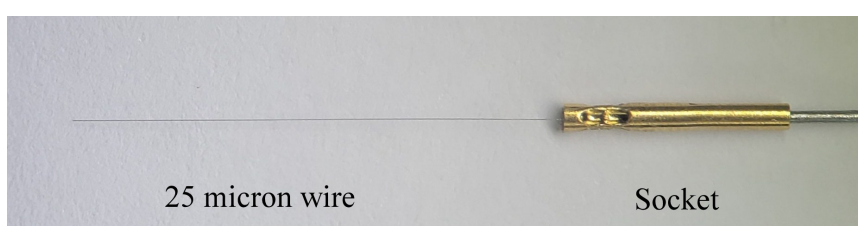

(a)

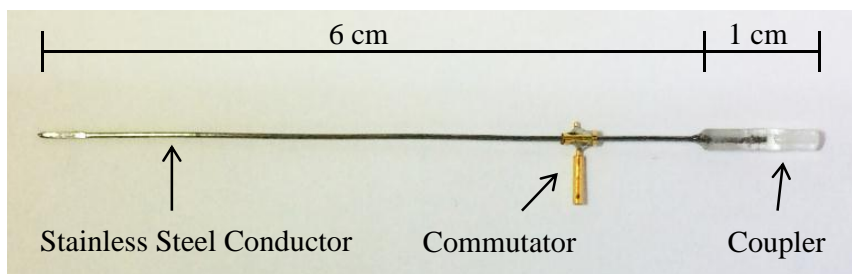

(b)

Fig. 2: (a) $25 \mu \mathrm{m}$ Pt microelectrode; (b) Electrode holder.

tube before coating to prevent it from being insulated. The typical thickness of parylene coating is $2 \mu \mathrm{m}$. Finally, the tip of the micro-wire is trimmed using fine scissors (Fine Science Tools, Foster City, CA, USA, Model: 15000-11) to expose Pt for sensing neural activity. The electrochemical impedance of the fabricated microelectrode is in the range of $0.5-1 \mathrm{M} \Omega$ at 1 $\mathrm{kHz}$ frequency. Fig. 2a shows a fabricated Pt microelectrode.

\section{B. Microelectrode Inserter Design}

This section presents a design of the motorized electrode inserter required to insert the microelectrode in the proposed method. Fig. 3a-3b show a 3D model of the inserter designed in Autodesk Fusion 360. The proposed electrode inserter consists of 4 major parts: digital Vernier caliper, insertion motor, spinner motor and the electrode guide. The last 3 parts are mounted on a digital Vernier scale obtained from AccuRemote, San Clemente, CA, USA (Part Number: 35812). The mounting parts are $3 \mathrm{D}$ printed. The insertion motor is a variable speed DC gear motor (obtained from Uxcell, Kwai Fong, Hong Kong, Part Number: a14052900ux0148) that drives the electrode up and down. Linear motion is obtained by using a $100 \mathrm{~mm}$ long and $6 \mathrm{~mm}$ diameter lead screw with $1 \mathrm{~mm}$ pitch. The spinner motor is a variable speed DC servo motor (obtained from FEETECH RC Model Co., Ltd., Part Number: FS90R) that spins the electrode during insertion. The electrode is attached to the spinner motor shaft using a holder shown in Fig. 2b. The electrode holder is made of a 0.5 $\mathrm{mm}$ diameter stainless steel conductor with a non-conductive motor shaft coupler. The other end of the holder plugs into the electrode socket. A custom-made socket, as shown in Fig. $2 \mathrm{~b}$, is used as a commutator. The insertion motor is attached to the fixed frame of the Vernier caliper and the spinner motor is attached to the movable Vernier scale. The insertion motor drives the Vernier scale and the readout indicates the position of the electrode.

The electrode guide, which is a key part of the proposed inserter, is located at the bottom of the frame (Fig. 3). The guide is demonstrated in Fig. 4 by inserting an electrode into agar gel. The top 3 pairs of arms are semi-flexible so that when the socket pushes them downward, they bend and the socket

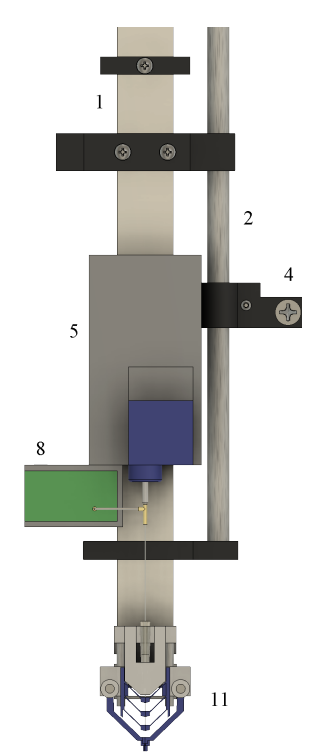

(a)

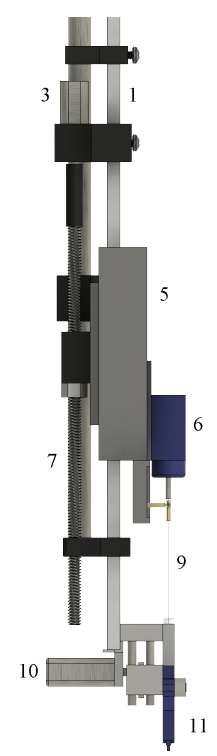

(b)

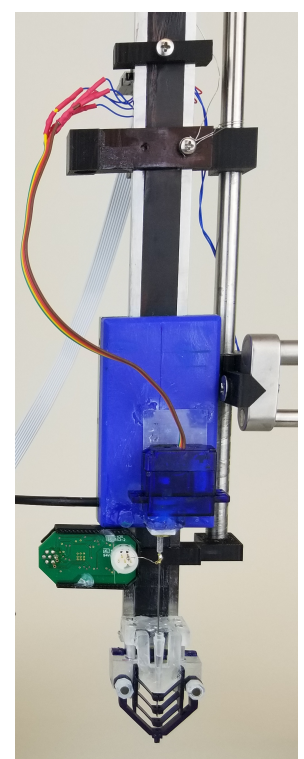

(c)
Fig. 3: A 3D model of the microelectrode inserter; (a) Front view; (b) Side view; (c) Actual device. [1- Fixed Frame, 2Support Rod, 3- Insertion Motor, 4- Stereotaxic Arm Clamp, 5-Movable Vernier Scale, 6- Spinner Motor, 7- Lead Screw, 8- Neural Amplifier Circuit Board, 9- Electrode Holder, 10Guide Lifting Motor, 11- Electrode Guide]

can pass through. The bottom arms are rigid but they can move upward and open up to release the socket after insertion. Fig. $4 \mathrm{a}$ shows the state of the guide and the position of the electrode before insertion. The rigid arms along with the lower two pairs of the semi-flexible arms are closed and surround the electrode wire. The top semi-flexible arms are open because of the electrode socket. The electrode is spun and slowly inserted into the agar gel through the guide. While inserting the electrode, the semi-flexible arms open up one by one as the electrode socket pushes them downward. After insertion, the electrode is in the agar gel and the electrode socket is above the rigid arms (Fig. 4b). The rigid arms are then retracted, as shown in Fig. 4c, to release the electrode. Fig. 4d shows a computer tomography (CT) image of the microelectrode inserted $10 \mathrm{~mm}$ in agar gel. The electrode appears as a thin white line in the image. The semi flexible arms are attached to the fixed frame but the rigid arms are mounted on a support which can slide up and down along the fixed frame. The up-down movement of the rigid arms are controlled by a motorized cam-wheel system shown in Fig. 4e and Fig. 4f. The cam-wheel is designed to provide $4 \mathrm{~mm}$ vertical displacement with $180^{\circ}$ rotation. While retracting the rigid arms, the extruded rigid frame (Fig. 4f) restricts the motion of the rigid arms and forces them to rotate. In this way the rigid arms open up to make space for fastening the electrode during in-vivo experiments without dislodging the electrode. At the ends of the arms, there are micro-holes each with the shape of a half cone, so that when two arms touch end to end, they make a complete conical micro-hole as shown in Fig. 5a. In addition to the conical micro-hole, the 
rigid arms, when closed together, create a $3 \mathrm{~mm}$ long microferrule below the conical hole (Fig. 5b). This particular guide design ensures perpendicular penetration in the brain, restricts the electrode from buckling during insertion, and minimizes friction between the electrode and the guide, thus providing better insertion force. The height of the micro-ferrule including the micro-hole is $4 \mathrm{~mm}$ and the rigid arms retract by $2 \mathrm{~mm}$ before opening. Therefore, the electrode has to be $6 \mathrm{~mm}$ longer than the depth of insertion. The electrode guide is fabricated in a high resolution $(10 \mu \mathrm{m})$ Ember SLA 3D printer from Autodesk.

A neural amplifier is attached to the movable Vernier scale. Neural signal from the electrode reaches the amplifier through the electrode holder and commutator. The amplifier is a second order Butterworth active filter with a gain of 1000 and a pass band of 200-3500 Hz. The amplified signal is then recorded. The fabricated microelectrode inserter is shown in Fig. 3c.

\section{IN-VIVO EXPERIMENTS}

To evaluate different rotational speeds and insertion speeds, electrodes were inserted at 1,2 and 3 revolution per second (rps) rotational speeds and at 100, 200 and $400 \mu \mathrm{m} / \mathrm{s}$ insertion speeds in two separate rat brains. Each combination of rotational and insertion speeds was evaluated by 6 trials. The $1 \mathrm{rps}$ rotational speed has 1, 2 and 5 failures when inserted at 100, 200 and $400 \mu \mathrm{m} / \mathrm{s}$ insertion speeds, respectively. The $2 \mathrm{rps}$
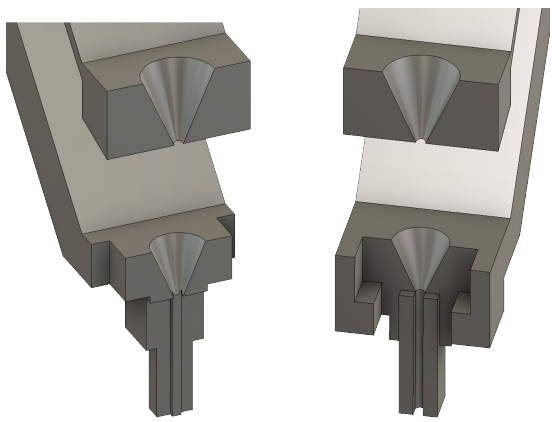

(a)

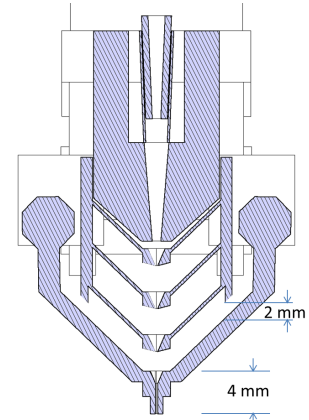

(b)
Fig. 5: (a) Conical holes at the ends of the arms. (b) Cross sectional view of the guide at mid-plane. rotational speed has 0,0 and 2 failures when inserted at 100 , 200 and $400 \mu \mathrm{m} / \mathrm{s}$, respectively. The $3 \mathrm{rps}$ rotational speed has 0 failure at all three insertion speeds. These results show that higher rotational speed allows faster insertion without failure. However, slow insertion allows blood vessels and brain tissue to accommodate the electrode [31] and, therefore, it is preferable. Thus, electrodes were inserted at $200 \mu \mathrm{m} / \mathrm{s}$ in the following experiments. Since 2 rps rotational speed showed 0 failure at $200 \mu \mathrm{m} / \mathrm{s}$ insertion speed, electrodes were inserted at that rotational speed. Slower rotational speed may result in insertion failure.

Microelectrodes were inserted in 10 non-survival experiments and 2 survival (chronic) experiments under anesthesia. All surgical and animal handling procedures were performed in accordance with the regulations of the Institutional Animal Care and Use Committee (IACUC) and approved by the Purdue University Animal Care and Use Committee (PACUC). Female Long Evans rats (250-274 gm) were anesthetized with Isoflurane (5\% for induction and $0.5-3 \%$ for maintenance, both in $2 \mathrm{~L} / \mathrm{min} \mathrm{O}_{2}$ ). The rat was mounted on a standard stereotaxic frame using ear bars and a nose cone. Butorphanol $(0.5-2 \mathrm{mg} / \mathrm{kg}, \mathrm{SQ})$ analgesic was injected. The surgical site was shaved and cleaned with $10 \%$ betadine and alcohol swabs.

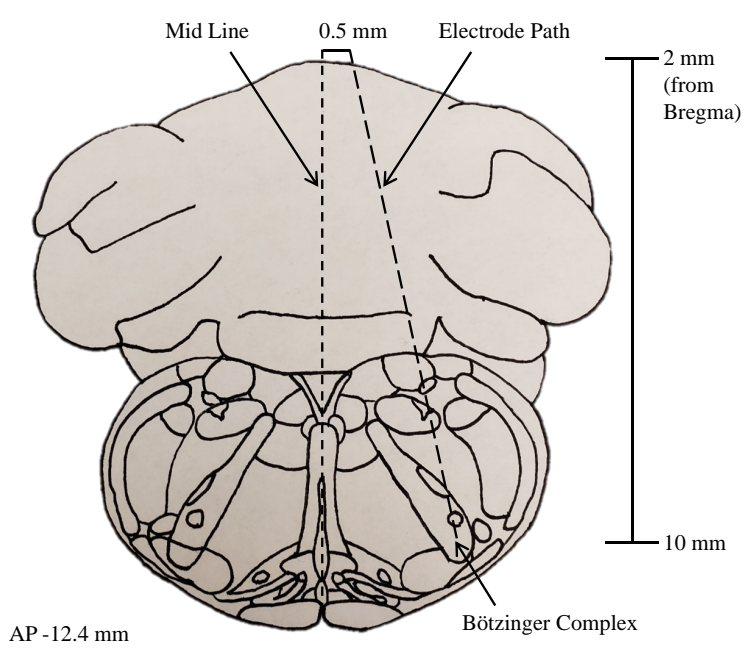

Fig. 6: A rat brain section at $12.4 \mathrm{~mm}$ behind bregma showing the location of the 'BötC' and the electrode insertion path. 
A mid-line incision was made on the skull. The 'Bötzinger complex (BötC)' of the brainstem was selected as the target of insertion. The 'BötC' is a group of neurons located in the rostral ventrolateral medulla and ventral respiratory column [32]. In rat, the 'BötC' is situated at $\sim 12.4 \mathrm{~mm}$ posterior to bregma, $\sim 2.2 \mathrm{~mm}$ lateral to mid-line and about $10 \mathrm{~mm}$ deep in the brain with reference to bregma as shown in Fig. 6 [33]. The complex spans only about $0.5 \mathrm{~mm}$ in the coronal plane. To avoid penetrating the sigmoid sinuses (large blood vessels), the electrode was inserted at an angle of $10^{\circ}$ to midline. Therefore, a $2 \mathrm{~mm}$ craniotomy hole was made in the skull at $0.5 \mathrm{~mm}$ lateral to mid-line instead of $2.2 \mathrm{~mm}$. The dura was left intact. A ALM self-retaining retractor was used to hold tissues and skin during surgery. The reason behind selecting the 'BötC' as the target is that it is located deep in the brain and the behavior of these neurons is known. The 'BötC' consists of respiratory neurons which generate inspiratory and expiratory activities [34]-[36]. The relationship between the neural activity recorded from electrode during insertion and the breathing of the rat provides a physiological marker for the target structure.

Now the inserter was attached to the stereotaxic arm for electrode insertion. An electrode was attached to the holder and loaded in the inserter. The electrode was positioned at the end of the micro-ferrule. The ferrule end was positioned on the craniotomy. In preparation for insertion, the spinner motor was accelerated to a speed of $2 \mathrm{rps}$. The spinning microelectrode was then advanced into the brain at a speed of $200 \mu \mathrm{m} / \mathrm{s}$. The depth of insertion was read from the digital Vernier scale. Both motions were turned off when the electrode went about $8 \mathrm{~mm}$ inside the brain. The electrode was then advanced in microsteps without spinning until a suitable neuron was found. A 3 -electrode recording system was used. The retractor was used as the reference and a subcutaneous injection needle in the abdominal cavity was used as the amplifier ground.

For chronic experiments, after reaching the target, the electrode was fixed on the skull as shown in Fig. 7. First, the rigid arms of the guide were retracted and they opened up without dislocating the electrode. Silicone sealant and UV cured dental cement were applied to fix the electrode to the skull. The electrode holder was retracted and detached from the electrode socket and the inserter was removed. Three stainless steel skull screws were implanted and connected by a wire with a pin to make the reference electrode. Finally, a plastic pedestal with holes was mounted on the skull to secure the microelectrode along with the reference electrode and the rat was recovered from anesthesia. Recordings were made under light anaesthesia various times after recovery from implantation surgery. Neural signal was obtained by connecting the amplifier to the electrodes with a cable. A subcutaneous injection needle in the abdominal cavity was used as the amplifier ground. After recording, the needle was removed and the rat was recovered from anesthesia.

\section{RESULTS}

Fig. 8 shows the neural activity recorded at progressive depths of insertion in a survival experiment. Various neural

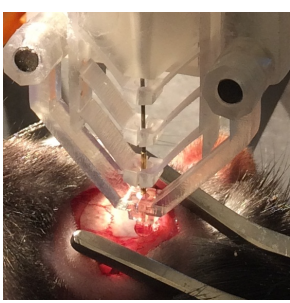

(a)

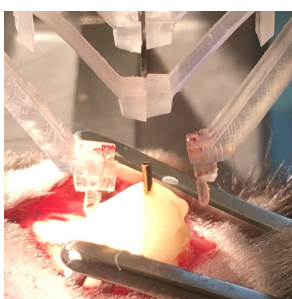

(d)

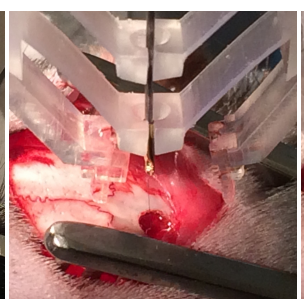

(b)

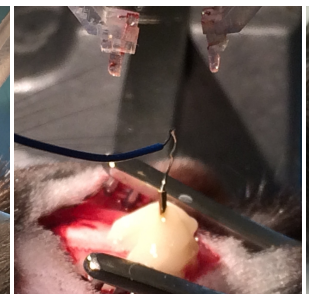

(e)

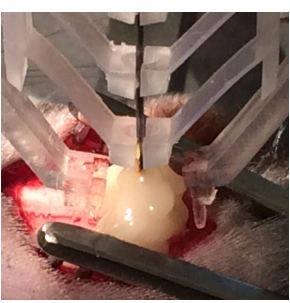

(c)

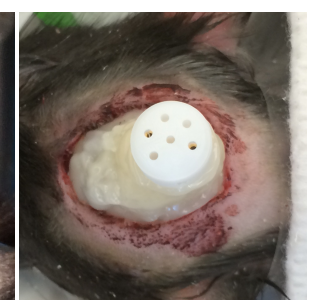

(f)
Fig. 7: In-vivo demonstration of the electrode fixing process in a survival experiment. (a) Microelectrode inserted in the brainstem of a rat. (b) Opening of rigid arms after insertion. The part of electrode above the brain is visible. (c) Application of dental cement. (d) Holder detached from electrode socket. (e) Recording cable plugged into socket to verify neural activity. (f) Plastic pedestal mounted on skull to secure electrode.

activities were observed at different positions of the electrode in the brain. A thermocouple was placed in front of the nose to sense the respiration. For each breathing cycle, the thermocouple generated a voltage oscillation due to the temperature variations in the inhaled and exhaled air. This respiratory signal is also shown in Fig. 8. The neural signal was played through a speaker and the rat's chest movements were observed. At $9.68 \mathrm{~mm}$ depth, neural firing was found to be correlated with the respiratory signal. The microelectrode was stepped further into the brain and a stronger respiratory neural activity was observed at $9.83 \mathrm{~mm}$. These observations demonstrated that the electrode reached the target region. Fig. 8 also shows the neural signal recorded at each phase of the electrode fixing process. The peri-event time histogram (PETH) of the detected spikes also shows the synchronization of the neural firing with respiration. Each PETH was obtained by averaging the spike rates from 20 respiratory cycles. The negative peak of each respiratory cycle was used to synchronize the spikes. Average cycle duration was used for segmenting the signal around the negative peaks. In survival experiments, neural activity was recorded for up to 50 days. Fig. 9 shows the neural signal recorded from the rat on day 1, 8, 21, 34 and 50. The spikes were sorted and the first three dominant spike groups are shown along with their PETHs. The first dominant unit is synchronized with respiration but the second and third units are not correlated with respiration. After 50 days post implantation, the rat was euthanized. To visualize the implanted microelectrode inside the brain, the rat head was scanned in a micro-CT scanner after perfusion [37]. The x-ray $3 \mathrm{D}$ image of the implanted microelectrode inside the brain is 

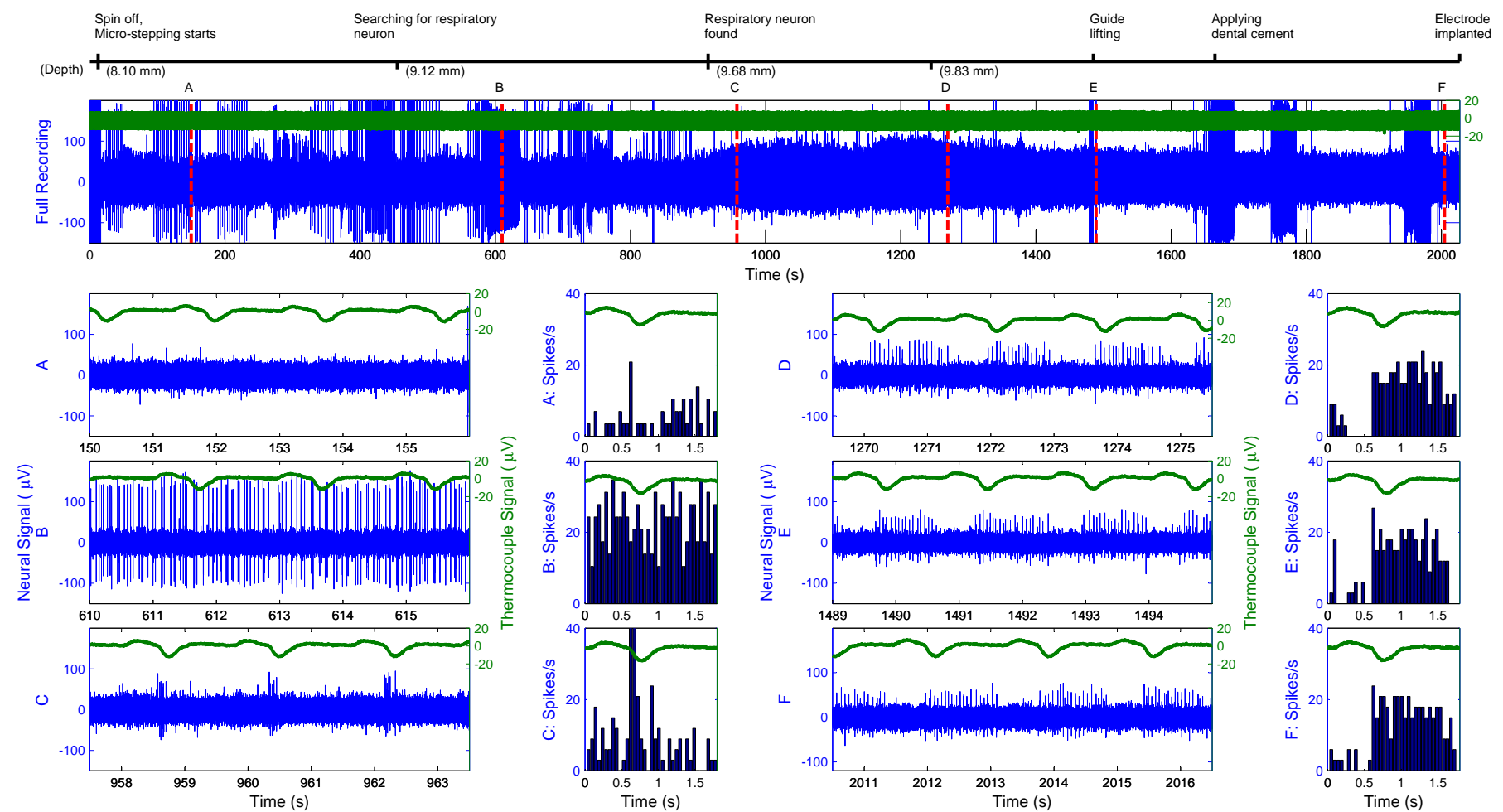

Fig. 8: Neural recordings at different steps of electrode insertion. Blue trace is the neural signal and green trace is the respiration signal. Each negative peak in the respiration signal represents an exhalation. Top plot: Full recording from micro-stepping to electrode implantation. The line at top shows the time stamps and associated electrode depth at various events during the insertion process. Plot A-F in the first and third columns show segments of the full recording at instances marked with red dash line on the full recording and the corresponding PETHs of the neural firing in a breathing cycle are shown in the second and fourth columns. Plot A: Recording at $8.40 \mathrm{~mm}$ depth during micro-stepping. Few small spikes are observed. Plot B: Recording at $9.32 \mathrm{~mm}$ depth. Many large spikes, although not related to respiration, are observed. Plot C: Appearance of the respiratory neural activity. Neural firing synchronized with respiration is observed at $9.68 \mathrm{~mm}$ depth. Plot D: Stronger respiratory neural activity observed at $9.83 \mathrm{~mm}$ depth. Plot E: Neural signal after opening the rigid arms. Plot F: Neural signal after applying dental cement.

shown in Fig. 10. The threshold for image reconstruction is set such that the brain tissue does not appear after reconstruction. The $25 \mu \mathrm{m}$ electrode appears thicker than its actual diameter because of artifacts. The electrode shows no signs of buckling in the brain. In all other 11 experiments, the electrode went into the brain without buckling. Respiratory neural spikes were observed in all rats except one. In that single acute experiment, we did not observe spikes synchronized with respiration. We believe that we missed the small target structure (BötC) due to the variability of brain structure between rats. The respiratory neural recordings from 3 non-survival experiments are shown in Fig. 11.

\section{Discussion}

Successful electrode insertion requires a straight electrode. A crimped or damaged electrode has a high probability of failure because of reduced CBF. In the in-vivo experiments, dura is not retracted prior to insertion. Although a higher insertion force is required to penetrate dura [38], [39], the electrode pierces dura by its spinning motion. The driving force for the electrode is the pressure applied to it. When the electrode touches brain surface, it initially creates a small dimple, unlike with impact insertion [40]. The electrode then slides inside the brain gradually. Fast insertion requires the electrode to withstand a greater insertion force which is not amenable to flexible structures. If the insertion happens very fast, the $25 \mu \mathrm{m} \mathrm{Pt}$ electrode can buckle with a large transverse displacement and since CBF decreases with increasing transverse displacement [41], the insertion can fail. The proposed insertion method is applicable for other electrode materials such as platinumiridium, tungsten, carbon fiber and stainless steel. Moreover, higher $\mathrm{CBF}$ is achievable by reducing the separation between the guide arms by which micro-wires thinner than $25 \mu \mathrm{m}$ can be inserted without buckling. Since the insertion process involves spinning the electrode, the technology is limited to cylindrical shaped micro-wire electrode. It does not work for planar or bed of needle array electrodes. Also the proposed method is not applicable to electrode probes made of soft materials like silicone, parylene or PDMS.

\section{CONCLUSION}

A novel flexible microelectrode insertion method is proposed and demonstrated. An electrode insertion device based on the proposed method also is presented. The device is 

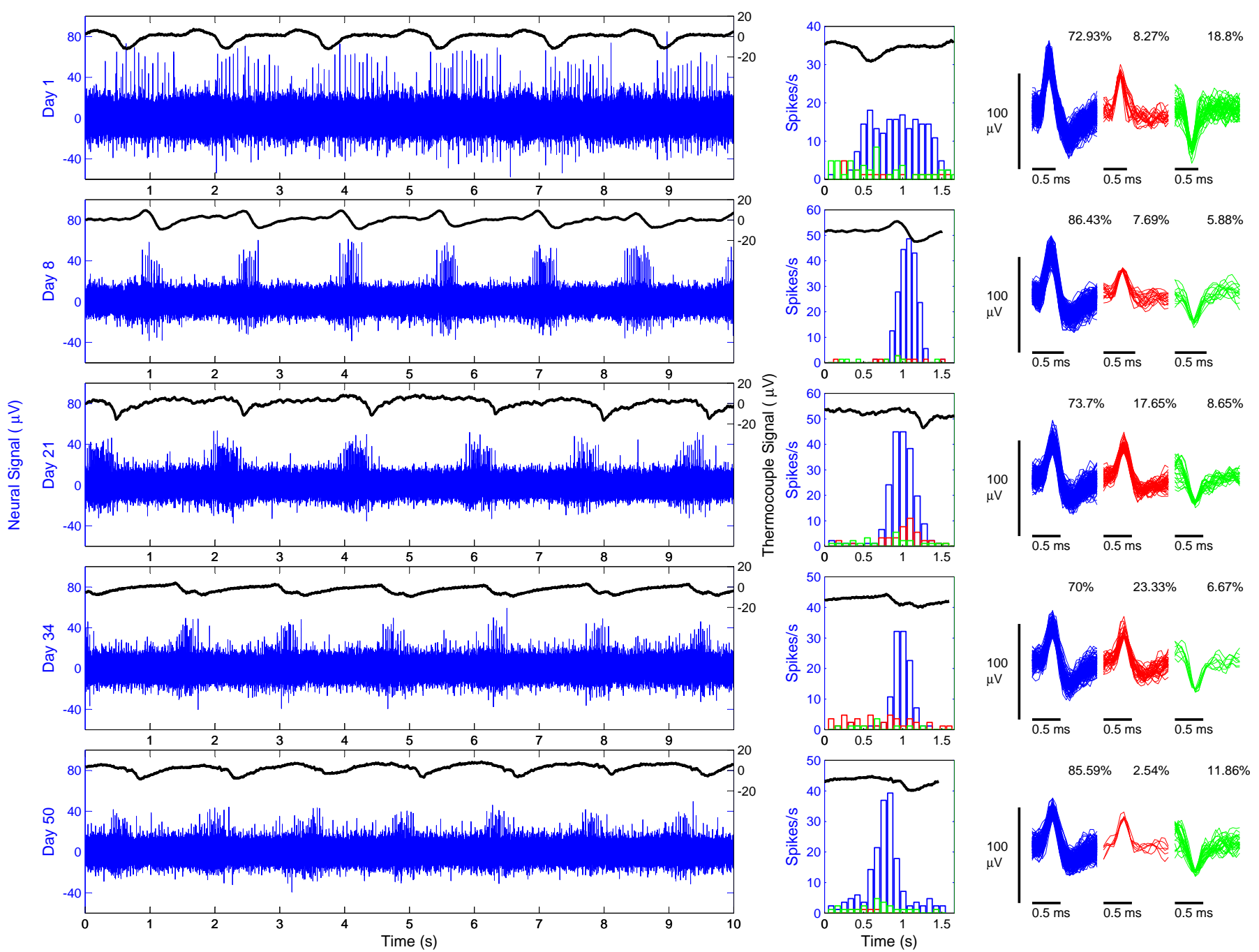
$73.7 \% \quad 17.65 \% \quad 8.65 \%$

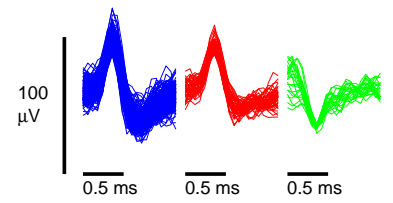
$70 \% \quad 23.33 \% \quad 6.67 \%$

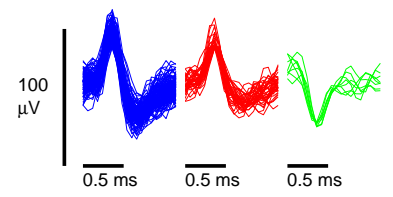
$2.54 \% \quad 11.86 \%$

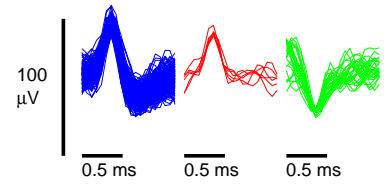

Fig. 9: Long-term neural recording from the Bötzinger Complex of rat. (Left) Segments of respiratory neural signal recorded on day 1, 8, 21, 34 and 50. Blue trace is the neural signal and black trace is the respiration signal. (Right) The 3 largest populated spike groups after spike sorting. The percentage number of spikes in each group are given. (Middle) The PETH of the three dominant spikes in a breathing cycle.

portable and battery operated. In-vivo experiments demonstrated successful insertions evidenced by electrophysiological recordings and $\mathrm{x}$-ray images. By spinning the electrode during insertion and inserting it through an appropriate electrode guiding system, it is possible to implant 25 micron wire electrodes in deep brain regions of rodents without any buckling and, therefore, accurately target specific neurons, in this case in the brainstem. We demonstrate successful implantations of 25 micron Pt flexible electrodes about $10 \mathrm{~mm}$ (with reference to bregma) deep in the brain without using any supporting tube, cannula or biodegradable coating and hence the method is novel. Since the electrode is inserted without piercing the brain with a rigid support, the method is less invasive. Electrodes were implanted in the Bötzinger complex of rat brains and the respiratory neural activity was observed up to 50 days post implantation. Therefore, the proposed method and the insertion device can be used to implant flexible microelectrodes in the deep brain structure of rodents for chronic neural recording.

\section{ACKNOWLEDGMENT}

The authors would like to thank Asem Aboelzabah and David McMillan, Department of Biomedical Engineering, Purdue University, for their help in the fabrication of the device. The authors would also like to thank Pamela Lachcik, Department of Nutrition Science, Purdue University, for her help in the CT scanning of the brain.

\section{REFERENCES}

[1] C. Im and J.-M. Seo, "A review of electrodes for the electrical brain signal recording," Biomed. Eng. Lett., vol. 6, no. 3, pp. 104-112, 2016.

[2] K. M. Szostak, L. Grand, and T. G. Constandinou, "Neural interfaces for intracortical recording: Requirements, fabrication methods, and characteristics," Front Neurosci, vol. 11, p. 665, 2017.

[3] P. Fattahi, G. Yang, G. Kim, and M. R. Abidian, "A review of organic and inorganic biomaterials for neural interfaces," Adv Mater, vol. 26, no. 12, pp. 1846-85, 2014.

[4] M. Jorfi, J. L. Skousen, C. Weder, and J. R. Capadona, "Progress towards biocompatible intracortical microelectrodes for neural interfacing applications," J Neural Eng, vol. 12, no. 1, p. 011001, 2015. 


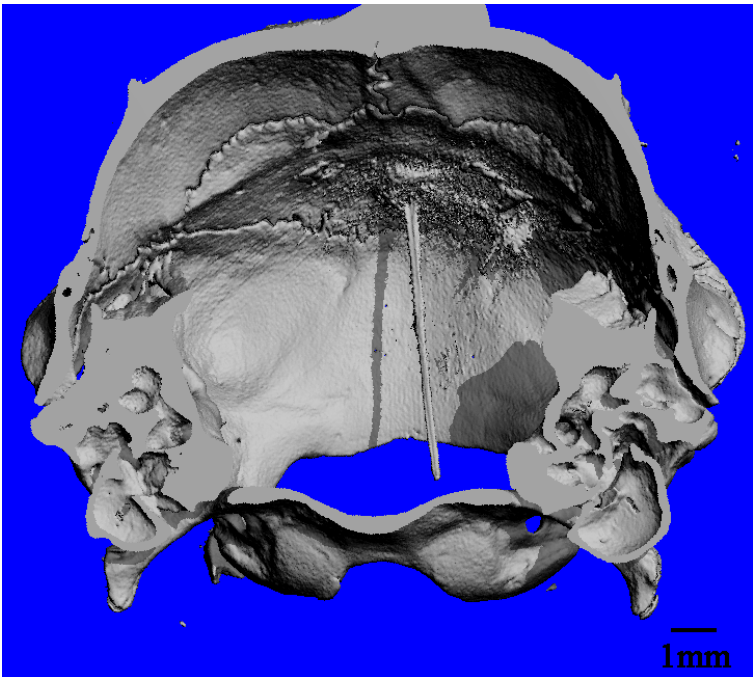

Fig. 10: x-ray 3D image of the microelectrode in rat brain.
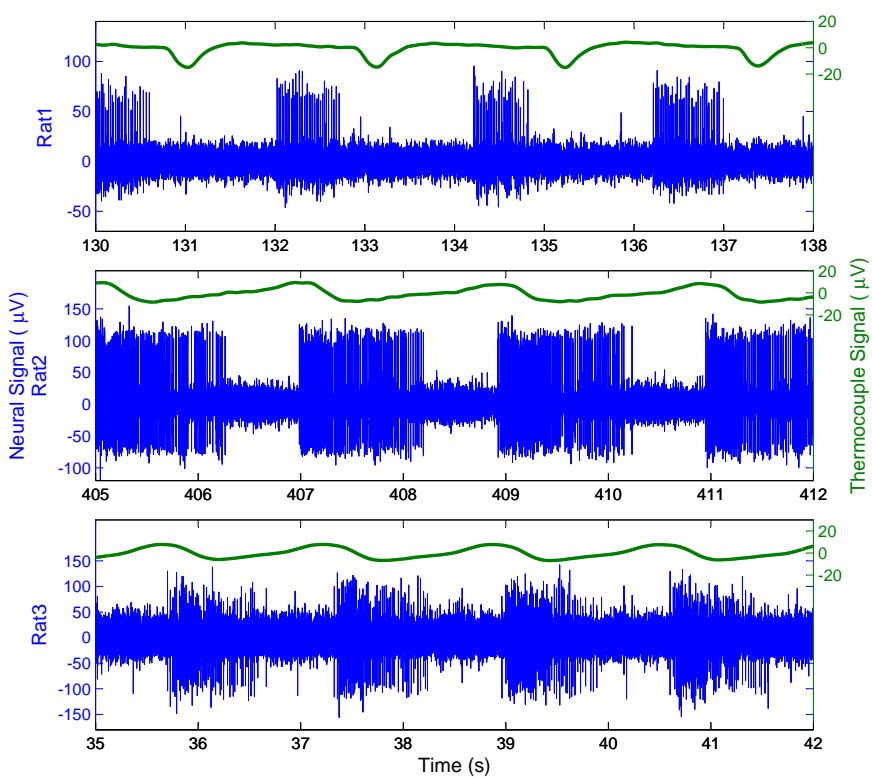

Fig. 11: Respiratory neural activity recorded from 3 nonsurvival experiments.

[5] A. C. Patil and N. V. Thakor, "Implantable neurotechnologies: a review of micro- and nanoelectrodes for neural recording," Medical \& Biological Engineering \& Computing, vol. 54, no. 1, pp. 23-44, Jan 2016.

[6] M. A. L. Nicolelis, "Brain-machine interfaces to restore motor function and probe neural circuits," Nat Rev Neurosci, vol. 4, no. 5, pp. 417-422, 2003.

[7] J. A. Gallego, M. G. Perich, L. E. Miller, and S. A. Solla, "Neural manifolds for the control of movement," Neuron, vol. 94, no. 5, pp. 978-984, 2017.

[8] P. Kennedy, "Changes in emotional state modulate neuronal firing rates of human speech motor cortex: a case study in long-term recording," Neurocase, vol. 17, no. 5, pp. 381-93, 2011.

[9] A. M. Lozano, A. E. Lang, R. Levy, W. Hutchison, and J. Dostrovsky, "Neuronal recordings in parkinson's disease patients with dyskinesias induced by apomorphine," Ann Neurol, vol. 47, no. 4 Suppl 1, pp. S1416, 2000.

[10] J. Martinerie, C. Adam, M. Le Van Quyen, M. Baulac, S. Clemenceau, B. Renault, and F. J. Varela, "Epileptic seizures can be anticipated by non-linear analysis," Nat Med, vol. 4, no. 10, pp. 1173-6, 1998.

[11] K. Smith, "Brain waves reveal intensity of pain," Nature, vol. 450, p 329,2007
[12] R. Biran, D. C. Martin, and P. A. Tresco, "Neuronal cell loss accompanies the brain tissue response to chronically implanted silicon microelectrode arrays," Exp Neurol, vol. 195, no. 1, pp. 115-26, 2005.

[13] G. Lind, C. E. Linsmeier, and J. Schouenborg, "The density difference between tissue and neural probes is a key factor for glial scarring," $S c i$ Rep, vol. 3, p. 2942, 2013.

[14] J. N. Turner, W. Shain, D. H. Szarowski, M. Andersen, S. Martins, M. Isaacson, and H. Craighead, "Cerebral astrocyte response to micromachined silicon implants," Exp Neurol, vol. 156, no. 1, pp. 33-49, 1999.

[15] D. H. Szarowski, M. D. Andersen, S. Retterer, A. J. Spence, M. Isaacson, H. G. Craighead, J. N. Turner, and W. Shain, "Brain responses to micromachined silicon devices," Brain Res, vol. 983, no. 1-2, pp. 23-35, 2003.

[16] V. S. Polikov, P. A. Tresco, and W. M. Reichert, "Response of brain tissue to chronically implanted neural electrodes," J Neurosci Methods, vol. 148 , no. 1, pp. 1-18, 2005.

[17] G. C. McConnell, H. D. Rees, A. I. Levey, C. A. Gutekunst, R. E. Gross, and R. V. Bellamkonda, "Implanted neural electrodes cause chronic, local inflammation that is correlated with local neurodegeneration," $J$ Neural Eng, vol. 6, no. 5, p. 056003, 2009.

[18] R. Biran, D. C. Martin, and P. A. Tresco, "The brain tissue response to implanted silicon microelectrode arrays is increased when the device is tethered to the skull," J Biomed Mater Res A, vol. 82, no. 1, pp. 169-78, 2007.

[19] T. D. Kozai, N. B. Langhals, P. R. Patel, X. Deng, H. Zhang, K. L. Smith, J. Lahann, N. A. Kotov, and D. R. Kipke, "Ultrasmall implantable composite microelectrodes with bioactive surfaces for chronic neural interfaces," Nat Mater, vol. 11, no. 12, pp. 1065-73, 2012.

[20] J. P. Seymour and D. R. Kipke, "Neural probe design for reduced tissue encapsulation in cns," Biomaterials, vol. 28, no. 25, pp. 3594-607, 2007.

[21] J. P. Harris, A. E. Hess, S. J. Rowan, C. Weder, C. A. Zorman, D. J. Tyler, and J. R. Capadona, "In vivo deployment of mechanically adaptive nanocomposites for intracortical microelectrodes," J Neural Eng, vol. 8, no. 4, p. 046010, 2011.

[22] S. P. Marshall, W. C. Lin, P. R. Patel, A. J. Shih, and C. A. Chestek, "Effects of geometry and material on the insertion oi very small neural electrode," 2016 38th Annual International Conference of the IEEE Engineering in Medicine and Biology Society (EMBC), pp. 2784-2788, 2016.

[23] S. Timoshenko, Theory of elastic stability. New York: Mc Graw-Hill, 1936.

[24] B. A. Wester, R. H. Lee, and M. C. LaPlaca, "Development and characterization of in vivo flexible electrodes compatible with large tissue displacements," J Neural Eng, vol. 6, no. 2, p. 024002, 2009.

[25] A. A. Sharp, A. M. Ortega, D. Restrepo, D. Curran-Everett, and K. Gall, "In vivo penetration mechanics and mechanical properties of mouse brain tissue at micrometer scales," IEEE Trans Biomed Eng, vol. 56, no. 1, pp. 45-53, 2009.

[26] W. Jensen, K. Yoshida, and U. G. Hofmann, "In-vivo implant mechanics of flexible, silicon-based acreo microelectrode arrays in rat cerebral cortex," IEEE Trans Biomed Eng, vol. 53, no. 5, pp. 934-40, 2006.

[27] T. J. Hall, M. Bilgen, M. F. Insana, and T. A. Krouskop, "Phantom materials for elastography," IEEE Transactions on Ultrasonics, Ferroelectrics, and Frequency Control, vol. 44, no. 6, pp. 1355-1365, 1997.

[28] T. I. Kim, J. G. McCall, Y. H. Jung, X. Huang, E. R. Siuda, Y. Li, J. Song, Y. M. Song, H. A. Pao, R. H. Kim, C. Lu, S. D. Lee, I. S. Song, G. Shin, R. Al-Hasani, S. Kim, M. P. Tan, Y. Huang, F. G. Omenetto, J. A. Rogers, and M. R. Bruchas, "Injectable, cellular-scale optoelectronics with applications for wireless optogenetics," Science, vol. 340, no. 6129, pp. 211-6, 2013.

[29] A. Williamson, M. Ferro, P. Leleux, E. Ismailova, A. Kaszas, T. Doublet, P. Quilichini, J. Rivnay, B. Rzsa, G. Katona, C. Bernard, and G. G. Malliaras, "Localized neuron stimulation with organic electrochemical transistors on delaminating depth probes," Adv Mater, vol. 27, no. 30, pp. 4405-4410, 2015.

[30] S. H. Felix, K. G. Shah, V. M. Tolosa, H. J. Sheth, A. C. Tooker, T. L. Delima, S. P. Jadhav, L. M. Frank, and S. S. Pannu, "Insertion of flexible neural probes using rigid stiffeners attached with biodissolvable adhesive," J Vis Exp, no. 79, p. e50609, 2013.

[31] K. J. Paralikar, J. K. Lawrence, and R. S. Clement, "Collagenaseaided insertion of intracortical microelectrode arrays: Evaluation of insertion force and chronic recording performance," 2006 International Conference of the IEEE Engineering in Medicine and Biology Society, pp. 2958-2961, 2006.

[32] E. G. Merrill, "Where are the real respiratory neurons?" Fed Proc, vol. 40, no. 9, pp. 2389-94, 1981.

[33] G. Paxinos and C. Watson, The rat brain in stereotaxic coordinates, 6th ed.tnAmsterdam ; Boston ; Oxford: Academic, 2006. 
1

2

3

3

4

5

6

7

8

9
[34] D. W. Richter, "Generation and maintenance of the respiratory rhythm," J Exp Biol, vol. 100, pp. 93-107, 1982.

[35] D. W. Richter and J. C. Smith, "Respiratory rhythm generation in vivo," Physiology (Bethesda), vol. 29, no. 1, pp. 58-71, 2014.

[36] T. H. Bryant, S. Yoshida, D. de Castro, and J. Lipski, "Expiratory neurons of the btzinger complex in the rat: a morphological study following intracellular labeling with biocytin," J Comp Neurol, vol. 335, no. 2, pp. 267-82, 1993.

[37] P. Sokal, M. Harat, M. Rusinek, M. Ruda, and A. Litwinowicz, "Intraoperative ct verification of electrode localization in dbs surgery in parkinson's disease," Interdisciplinary Neurosurgery, vol. 2, no. 1, pp. 6-9, 2015.

[38] Z. Fekete, A. Nmeth, G. Mrton, I. Ulbert, and A. Pongrcz, "Experimental study on the mechanical interaction between silicon neural microprobes and rat dura mater during insertion," J Mater Sci Mater Med, vol. 26, no. 2, p. 70, 2015.

[39] K. Najafi and J. F. Hetke, "Strength characterization of silicon microprobes in neurophysiological tissues," IEEE Trans Biomed Eng, vol. 37, no. 5, pp. 474-81, 1990.

[40] P. J. Rousche and R. A. Normann, "A method for pneumatically inserting an array of penetrating electrodes into cortical tissue," Ann Biomed Eng, vol. 20, no. 4, pp. 413-22, 1992.

[41] A. Zikowski and S. Imieowski, "Buckling and post-buckling behaviour of prismatic aluminium columns submitted to a series of compressive loads," Experimental Mechanics, vol. 51, no. 8, pp. 1335-1345, 2011. 